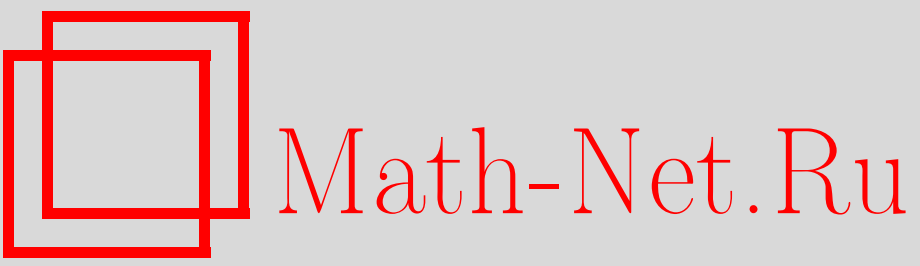

А. В. Псху, Функция Грина первой краевой задачи для дробного диффузионноволнового уравнения в многомерной прямоугольной области, Итоги науки и техн. Сер. Соврем. мат. и ее прил. Темат. обз., 2019, том 167, 52-61

DOI: https://doi.org/10.36535/0233-6723-2019-167-52-61

Использование Общероссийского математического портала Math-Net.Ru подразумевает, что вы прочитали и согласны с пользовательским соглашением

http://www.mathnet.ru/rus/agreement

Параметры загрузки:

IP: 54.162 .85 .209

26 апреля 2023 г., $16: 27: 26$ 


\title{
ФУНКЦИЯ ГРИНА ПЕРВОЙ КРАЕВОЙ ЗАДАЧИ ДЛЯ ДРОБНОГО ДИФФУЗИОННО-ВОЛНОВОГО УРАВНЕНИЯ В МНОГОМЕРНОЙ ПРЯМОУГОЛЬНОЙ ОБЛАСТИ
}

\author{
(C) 2019 г. $\quad$ A. В. ПСХУ
}

АннотАция. В работе построены функции Грина первой краевой задачи для дробного диффузионно-волнового уравнения в многомерных (ограниченных и неограниченных) гиперпрямоугольных областях.

Ключевъе слова: диффузионно-волновое уравнение, функция Грина, дробная производная, оператор Джрбашяна-Нерсесяна, краевая задача, условие А. Н. Тихонова.

\section{GREEN FUNCTION OF THE FIRST BOUNDARY-VALUE PROBLEM FOR THE FRACTIONAL DIFFUSION WAVE EQUATION IN A MULTIDIMENSIONAL RECTANGULAR DOMAIN}

\author{
(c) 2019 A. V. PSKHU
}

\begin{abstract}
In this paper, the Green functions of the first boundary-value problem for the fractional diffusion wave equation in multidimensional (bounded and unbounded) hyper-rectangular domains are constructed.
\end{abstract}

Keywords and phrases: diffusion wave equation, Green function, fractional derivative, DzhrbashyanNersesyan operator, boundary-value problem, Tikhonov condition.

AMS Subject Classification: 35R11, 35K 20

1. Введение. Рассмотрим уравнение

$$
\left(\frac{\partial^{\sigma}}{\partial y^{\sigma}}-\Delta_{x}\right) u(x, y)=f(x, y)
$$

где $\partial^{\sigma} / \partial y^{\sigma}$ - дробная производная порядка $\sigma$ по переменной $y$ с началом в точке $y=0 ; \sigma \in(0,2)$;

$$
\Delta_{x}=\sum_{k=1}^{n} \frac{\partial^{2}}{\partial x_{k}^{2}}
$$

- оператор Лапласа по переменной $x, x=\left(x_{1}, x_{2}, \ldots, x_{n}\right) \in S \subset \mathbb{R}^{n}$.

Уравнение (1) является уравнением диффузии при $\sigma=1$ и превращается в волновое уравнение при $\sigma=2$. Поэтому при $\sigma \in(0,2)$ уравнение (1) принято называть диффузионно-волновым уравнением. Это уравнение интенсивно исследовалось в последние десятилетия. Укажем в этой связи работы $[2,4,6-8,14,16-19,21,26,30]$, содержащие основные подходы к исследованию дробных диффузионных и диффузионно-волновых уравнений и более полную библиографию.

Работа выполнена при поддержке Российского фонда фундаментальных исследований (проект № 18-51-45005). 
Укажем, также, монографии $[5,9,12,20,23-25]$, посвященные теории дробного исчисления и дифференциальных уравнений дробного порядка.

Цель данной работы - построить функцию Грина краевой задачи для уравнения (1) в многомерной цилиндрической области, в основании которой лежит гиперпрямоугольная (ограниченная или неограниченная) область.

В одномерном случае функции Грина первой, второй и смешанных краевых задач были построены в $[10,11]$. Укажем также работу [1], в которой строится решение первой краевой задачи для уравнения диффузии в многомерной области.

Отметим, что интерес к дифференциальным уравнениям дробного порядка стимулируется, также, различными приложениями в физике и механике (см. $[5,13,15,22,27])$.

2. Дробное дифференцирование. Оператор Джрбашяна-Нерсесяна порядка $\sigma$ с началом в точке $y=\eta$, ассоциированный с последовательностью

$$
\left\{\gamma_{0}, \gamma_{1}, \ldots, \gamma_{m}\right\}, \quad 0<\gamma_{k} \leqslant 1, \quad \sigma=\gamma_{0}+\gamma_{1}+\cdots+\gamma_{m}-1
$$

(см. [3]) определяется соотношением

$$
\frac{\partial^{\sigma}}{\partial(y-\eta)^{\sigma}}=D_{\eta y}^{\left\{\gamma_{0}, \gamma_{1}, \ldots, \gamma_{m}\right\}} \equiv D_{\eta y}^{\gamma_{m}-1} D_{\eta y}^{\gamma_{m}-1} \ldots D_{\eta y}^{\gamma_{0}}
$$

где $D_{\eta y}^{\gamma_{m}-1}$ и $D_{\eta y}^{\gamma_{k}}$ - дробные интеграл и производные Римана-Лиувилля. Оператор дробного интегро-дифференцирования Римана-Лиувилля с началом в точке $y=\eta$ порядка $\gamma \in \mathbb{R}$ определяется равенствами

$$
\begin{gathered}
D_{\eta y}^{\gamma} h(y)=\frac{\operatorname{sign}(y-\eta)}{\Gamma(-\gamma)} \int_{\eta}^{y}|y-t|^{-\gamma-1} h(t) d t, \quad \gamma<0 ; \quad D_{\eta y}^{0} h(y)=h(y) ; \\
D_{\eta y}^{\gamma}=\operatorname{sign}^{n}(y-\eta) \frac{\partial^{p}}{\partial y^{p}} D_{\eta y}^{\gamma-p}, \quad p-1<\gamma \leqslant p, \quad p \in \mathbb{N} .
\end{gathered}
$$

Несложно заметить, что производная Римана-Лиувилля $D_{\eta y}^{\sigma}$ и производная Капуто $\partial_{\eta y}^{\sigma}$ являются частными случаями оператора Джрбашяна-Нерсесяна:

$$
D_{\eta y}^{\sigma}=D_{\eta y}^{\{\sigma-m+1,1, \ldots, 1\}}, \quad \partial_{\eta y}^{\sigma}=D_{\eta y}^{\{1,1, \ldots, 1, \sigma-m+1\}} .
$$

3. Область. Уравнение (1) будем рассматривать в цилиндрической области $\Omega$, имеющей основанием $n$-мерной гиперпрямоугольник, каждое из ребер которого есть отрезок, луч или прямая, а именно,

$$
\Omega=S \times(0, T)
$$

где

$$
S=\left(a_{1}, b_{1}\right) \times\left(a_{2}, b_{2}\right) \times \cdots \times\left(a_{n}, b_{n}\right), \quad-\infty \leqslant a_{k}<b_{k} \leqslant \infty, \quad k=\overline{1, n} .
$$

Таким образом, цилиндрическая область $\Omega$, как и ее основание $S$, определяется упорядоченным набором пар $\left\{\left(a_{1}, b_{1}\right), \ldots,\left(a_{n}, b_{n}\right)\right\}$; при этом некоторые из $a_{k}$ и $b_{k}$ могут принимать значения $-\infty$ и $+\infty$. Свяжем с этим набором подмножества $K_{i j}(i, j=0,1)$ множества $\{1,2, \ldots, n\}$, определяемые следующим образом:

$$
\begin{array}{ll}
K_{00}=\left\{k: a_{k}=-\infty, b_{k}=\infty\right\}, & K_{01}=\left\{k: a_{k}=-\infty, b_{k}<\infty\right\}, \\
K_{10}=\left\{k:-\infty<a_{k}, b_{k}=\infty\right\}, & K_{11}=\left\{k:-\infty<a_{k}, b_{k}<\infty\right\},
\end{array}
$$

т.е. $K_{00}$ - множество всех $k \in\{1,2, \ldots, n\}$, для которых вся ось $O x_{k}$ является ребром прямоугольника $S$. Множества $K_{01}$ и $K_{10}$ содержат все $k$, для которых ребро прямоугольника $S$, лежащее на оси $O x_{k}$, есть луч. Наконец, если $k \in K_{11}$, то соответствующее ребро является отрезком. Очевидно, что эти множества попарно не пересекаются, а их объединение дает $\{1,2, \ldots, n\}$.

Для всех $k \in K_{10} \cup K_{11}$ обозначим через $S_{k}^{a}$ гипергрань прямоугольника $S$, лежащую в гиперплоскости $x_{k}=a_{k}$, т.е. $S_{k}^{a}$-множество внутренних точек (в топологии $\mathbb{R}^{n-1}$ ) множества $\partial S \cap\left\{x_{k}=a_{k}\right\}$ (как обычно, $\partial S$ обозначает границу множества $S$ ). В случае, если $k \in K_{01} \cup K_{11}$, то $S_{k}^{b}$ - внутренние точки множества $\partial S \cap\left\{x_{k}=b_{k}\right\}$. 
4. Постановка задачи. Для точки $x \in \mathbb{R}^{n}, x=\left(x_{1}, x_{2}, \ldots, x_{n}\right)$, через $x_{(k)}$ будем обозначать ее проекцию на $\mathbb{R}^{n-1}$ вдоль $x_{k}: x_{(k)}=\left(x_{1}, \ldots, x_{k-1}, x_{k+1}, \ldots, x_{n}\right) \in \mathbb{R}^{n-1}$, и

$$
S_{(k)}=\left(a_{1}, b_{1}\right) \times \cdots \times\left(a_{k-1}, b_{k-1}\right) \times\left(a_{k+1}, b_{k+1}\right) \times \cdots \times\left(a_{n}, b_{n}\right) .
$$

Рассматривается следующая задача: найти регулярное решение уравнения

$$
\left(D_{0 y}^{\left\{\gamma_{0}, \ldots, \gamma_{m}\right\}}-\Delta_{x}\right) u(x, y)=f(x, y)
$$

в области $\Omega$, удовлетворяющее начальным условиям

$$
\lim _{y \rightarrow 0} D_{0 y}^{\left\{\gamma_{0}, \ldots, \gamma_{j}\right\}} u(x, y)=\tau_{j}(x), \quad x \in S, \quad j=0,1, \ldots, m-1,
$$

и краевым условиям

$$
\begin{array}{llll}
\left.u(x, y)\right|_{x_{k}=a_{k}}=\varphi_{k}\left(x_{(k)}, y\right), & k \in K_{10} \cup K_{11}, & x_{(k)} \in S_{(k)}, & y \in(0, T), \\
\left.u(x, y)\right|_{x_{k}=b_{k}}=\psi_{k}\left(x_{(k)}, y\right), & k \in K_{01} \cup K_{11}, & x_{(k)} \in S_{(k)}, & y \in(0, T) .
\end{array}
$$

Под регулярным решением уравнения (3) в области $\Omega$ мы подразумеваем такую функцию $u=u(x, y)$, что $y^{1-\mu} u(x, y) \in C(\bar{\Omega}), D_{0 y}^{\left\{\gamma_{0}, \ldots, \gamma_{j}\right\}} u(x, y) \in C(\Omega \cup\{x \in S, y=0\}), j=\overline{1, m-1}$; в области $\Omega$ функция $u(x, y)$ имеет непрерывные производные до второго порядка по каждой из переменных $x_{k}, k=\overline{1, n}$, а функции $D_{0 y}^{\left\{\gamma_{0}, \ldots, \gamma_{j}\right\}} u(x, y), j=\overline{1, m-1}$, непрерывно дифференцируемы по $y ; D_{0 y}^{\left\{\gamma_{0}, \ldots, \gamma_{m}\right\}} u(x, y) \in C(\Omega) ; u(x, y)$ удовлетворяет уравнению (3) во всех точках области $\Omega$.

Заметим, что для $k \in K_{11}$ область $S$ ограничена с двух сторон гипергранями $S_{k}^{a}$ и $S_{k}^{b}$, перпендикулярными оси $O x_{k}$. В этом случае на каждой гиперграни задаются условия, соответственно (5) и (6). Если $k \in K_{10} \cup K_{01}$, то область $S$ ограничена только с одной стороны вдоль $x_{k}$, и для этих индексов условия задаются либо на $S_{k}^{a}$, либо на $S_{k}^{b}$ (одно из условий (5) или (6)). Вдоль осей $O x_{k}$, для которых $k \in K_{00}$, множество $S$ неограниченно, и поэтому для этих индексов краевые условия отсутствуют. В частности, если множество $K_{10} \cup K_{11}\left(K_{01} \cup K_{11}\right)$ пусто, то условия (6) (условия (5)) не накладываются. Если же $K_{10} \cup K_{01} \cup K_{11}=\emptyset$, то задача ставится без условий (5) и (6).

5. Вспомогательные утверждения. Для произвольной функции $g=g(t)$, заданной на $\mathbb{R} \backslash\{0\}$, введем в рассмотрение оператор

$$
\mathbf{J}^{a, b} g=\left(\mathbf{J}^{a, b} g\right)(s, \theta)=\sum_{j=-\infty}^{\infty} g(s-\theta+2 j(b-a))-g(s+\theta-2 a+2 j(b-a)) .
$$

Относительно переменных и параметров $s, \theta, a$ и $b$ полагаем

$$
a<s, \theta<b, \quad s \neq \theta ; \quad-\infty \leqslant a<b \leqslant \infty .
$$

В случае, когда один или оба из параметров $a$ и $b$ принимают бесконечное значение, оператор (7) доопределяется следующим образом:

$$
\mathbf{J}^{a, b} g= \begin{cases}g(s-\theta)-g(s+\theta-2 a), & \text { если }-\infty<a, b=\infty, \\ g(s-\theta)-g(s+\theta-2 b), & \text { если } a=-\infty, b<\infty, \\ g(s-\theta), & \text { если } a=-\infty, b=\infty .\end{cases}
$$

Предполагается, что поведение функции $g(t)$ при $|t| \rightarrow \infty$ обеспечивает сходимость ряда в (7). Для наших целей достаточно потребовать существование предела

$$
\lim _{t \rightarrow \pm \infty}|t|^{\gamma} g(t)=0
$$

для некоторого $\gamma>1$. Если условие (9) выполнено, то ряд в (7), очевидно, сходится.

Далее для удобства будем писать $g(t) \in B_{\gamma}$, если функция $g(t)$ удовлетворяет условию (9). Также в дальнейшем буквой $C$ будем обозначать положительные константы, вообще говоря, разные в разных случаях, указывая в случае необходимости параметры, от которых они могут зависеть: $C=C(\alpha, \beta, \ldots)$. 
Лемма 1. Пусть $g(t) \in C^{p}(\mathbb{R} \backslash\{0\}), g^{(i)}(t) \in B_{\gamma}, i=\overline{0, p}, \gamma>1, p \in \mathbb{N} \cup\{0\}$. Тогда справедливъ соотношения

$$
\left(\mathbf{J}^{a, b} g\right)(s, \theta)=g(s-\theta)+H(s, \theta),
$$

где

$$
\begin{gathered}
H(s, \theta) \in C^{p}([a, b] \times(a, b)) \cap C^{p}((a, b) \times[a, b]) ; \\
\frac{\partial^{i}}{\partial s^{i}}\left(\mathbf{J}^{a, b} g\right)(s, \theta)=\left(\mathbf{J}^{a, b} g^{(i)}\right)(s, \theta), \quad i \leqslant p ; \\
\frac{\partial^{2 i}}{\partial \theta^{2 i}}\left(\mathbf{J}^{a, b} g\right)(s, \theta)=\left(\mathbf{J}^{a, b} g^{(2 i)}\right)(s, \theta), \quad 2 i \leqslant p .
\end{gathered}
$$

Доказательство. Если $a=-\infty$ или $b=\infty$, то включение (10), очевидно, следует из определения (8). Рассмотрим случай, когда $a>-\infty$ и $b<\infty$. Будем считать, что $s$ и $\theta$ могут изменяться в промежутках

$$
a+\delta_{1} \leqslant s \leqslant b-\delta_{1}, \quad a+\delta_{2} \leqslant \theta \leqslant b-\delta_{2}, \quad \text { где } \quad \delta_{1}, \delta_{2} \geqslant 0, \quad \delta=\max \left\{\delta_{1}, \delta_{2}\right\}>0 .
$$

Для $j \neq 0$ выражение $|s-\theta+2 j(b-a)|$ можно записать в виде

$$
|s-\theta+2 j(b-a)|=(2|j|-1)(b-a)+Q,
$$

где

$$
Q=(b-a)+(s-\theta) \operatorname{sign} j \geqslant b-\max \{s, \theta\}+\min \{s, \theta\}-a>\delta .
$$

Так как $\gamma>1$, отсюда следует, что

$$
|s-\theta+2 j(b-a)|^{\gamma} \geqslant(2|j|-1)^{\gamma}(b-a)^{\gamma}+\delta^{\gamma}, \quad j= \pm 1, \pm 2, \ldots .
$$

Аналогично преобразуем $|s+\theta-2 a+2 j(b-a)|$. Учитывая, что $s+\theta-2 a>\delta$, для $j \geqslant 0$ имеем

$$
|s+\theta-2 a+2 j(b-a)|^{\gamma} \geqslant[2 j(b-a)]^{\gamma}+\delta^{\gamma}, \quad j=0,1,2, \ldots
$$

Если же $j<0$, то

$$
|s+\theta-2 a+2 j(b-a)|=(2|j|-1)(b-a)+2 b-s-\theta \geqslant(2|j|-1)(b-a)+\delta,
$$

т.e.

$$
|s+\theta-2 a+2 j(b-a)|^{\gamma} \geqslant(2|j|-1)^{\gamma}(b-a)^{\gamma}+\delta^{\gamma}, \quad j=-1,-2, \ldots
$$

Из неравенств (13), (14) и (15), в силу (9), следует, что

$$
\left|\sum_{j=-\infty}^{\infty}[g(s-\theta+2 j(b-a))-g(s+\theta+2 j(b-a))]-g(s-\theta)\right| \leqslant C \sum_{i=0}^{\infty} \frac{1}{[i(b-a)]^{\gamma}+\delta^{\gamma}} .
$$

Это означает, что данный ряд сходится равномерно относительно любого замкнутого подмножества одного из множеств $(a, b) \times[a, b]$ или $[a, b] \times(a, b)$. Отсюда следует включение (10) при $p=0$.

Далее, условие $(9)$ для $g^{(i)}(t), i=0,1, \ldots, p$, гарантирует возможность дифференцировать под знаком суммы в (7). Отсюда следует справедливость (10) для $p>0$, а формулы (11) и (12) получаются почленным дифференцированием в (7) и (8).

Лемма 2. Пусть $g(t) \in C(\mathbb{R} \backslash\{0\}), g(t) \in B_{\gamma}, \gamma>1$. Тогда

$$
\lim _{\theta \rightarrow a+}\left(\mathbf{J}^{a, b} g\right)(s, \theta)=0, \quad \lim _{\theta \rightarrow b-}\left(\mathbf{J}^{a, b} g\right)(s, \theta)=0, \quad s \in(a, b) .
$$

Доказательство. Если $a=-\infty$ или $b=\infty$, то утверждение леммы следует непосредственно из (8) и (9). В случае, когда оба параметра $a$ и $b$ конечны, как было показано выше (см. доказательство леммы 1 ), условие (9) обеспечивает равномерную сходимость ряда в (7) на замкнутых подмножествах $(a, b) \times[a, b]$ или $[a, b] \times(a, b)$. Поэтому можно перейти к пределу под знаком суммы, т.е.

$$
\lim _{\theta \rightarrow a} \mathbf{J}^{a, b} g=\sum_{j=-\infty}^{\infty} \lim _{\theta \rightarrow a}[g(s-\theta+2 j(b-a))-g(s+\theta-2 a+2 j(b-a))]=0 .
$$


Также в случае $\theta \rightarrow b$

$$
\begin{aligned}
\lim _{\theta \rightarrow b} \mathbf{J}^{a, b} g=\sum_{j=-\infty}^{\infty} g(s-b+2 j(b-a))-g(s+b-2 a+2 j(b-a)) & = \\
& =\sum_{j=-\infty}^{\infty} g(s-b+2 j(b-a))-g(s-b+2(j+1)(b-a))=0 .
\end{aligned}
$$

Далее, для функции $g_{\lambda}(t)$, зависящей от параметра $\lambda \in \Lambda$, будем писать $g_{\lambda}(t) \in B_{\gamma}(\Lambda)$, если существует предел

$$
\lim _{t \rightarrow \pm \infty}|t|^{\gamma} g_{\lambda}(t)=0
$$

равномерный относительно $\Lambda$.

Лемма 3. Пусть $g_{\lambda}(t) \in C(\mathbb{R} \backslash\{0\})$, функиия $g_{\lambda}(t)$ зависит от параметра $\lambda \in \Lambda \subset \mathbb{R} u$ $g_{\lambda}(t) \in B_{\gamma}(\Lambda)$. Тогда справедливы следуюшие утверждения:

(1) если для любого $t \neq 0$ существует предел

$$
\lim _{\lambda \rightarrow \lambda_{0}} g_{\lambda}(t)=h(t)
$$

mo

$$
\lim _{\lambda \rightarrow \lambda_{0}}\left(\mathbf{J}^{a, b} g_{\lambda}\right)(s, \theta)=\left(\mathbf{J}^{a, b} h\right)(s, \theta) ;
$$

(2) если $g_{\lambda}(t)$ интегируема на $\left(\lambda_{0}, \lambda_{1}\right) \subset \Lambda$ как функиия $\lambda$ при каждом $t \neq 0$, то

$$
\int_{\lambda_{0}}^{\lambda_{1}}\left(\mathbf{J}^{a, b} g_{\lambda}\right)(s, \theta) d \lambda=\left(\mathbf{J}^{a, b} \int_{\lambda_{0}}^{\lambda_{1}} g_{\lambda} d \lambda\right)(s, \theta) ;
$$

(3) если $g_{\lambda}(t)$ дифференцируема по $\lambda$ при $t \neq 0$ и $\partial g_{\lambda}(t) / \partial \lambda \in B_{\gamma}$, то

$$
\frac{\partial}{\partial \lambda}\left(\mathbf{J}^{a, b} g_{\lambda}\right)(s, \theta)=\left(\mathbf{J}^{a, b} \frac{\partial}{\partial \lambda} g_{\lambda}\right)(s, \theta) .
$$

При этом сходимость в (17) и сходимость интеграла в (18) являются равномерными относительно в и $\theta$ из замкнутых подмножеств множеств

$$
(a, b) \times[a, b] \backslash\{s=\theta\} \quad \text { или } \quad[a, b] \times(a, b) \backslash\{s=\theta\} .
$$

Доказательство. Если $a=\infty$ или $b=\infty$, то утверждение леммы следует из (8). В случае, когда $a$ и $b$ конечны, так же, как и при доказательстве леммы 1 , можно показать, что ряд, определяющий $\mathbf{J}^{a, b} g_{\lambda}$ (за вычетом слагаемого $g_{\lambda}(s-\theta)$ ), сходится равномерно на замкнутых подмножествах множеств $(a, b) \times[a, b] \times \Lambda$ или $[a, b] \times(a, b) \times \Lambda$. Это обеспечивает возможность перехода к пределу под знаком суммы и почленного интегрирования соответствующих рядов и доказывает (17) и (18). Кроме того, вместе с условием $\partial g_{\lambda}(t) / \partial \lambda \in B_{\gamma}(\Lambda)$, это дает возможность дифференцировать под знаком суммы, и мы приходим к (19).

6. Фундаментальное решение. Рассмотрим функцию (см. [7])

$$
\Gamma_{\sigma, n}(x, y)=C_{n} y^{\beta(2-n)-1} f_{\beta}\left(|x| y^{-\beta} ; n-1, \beta(2-n)\right), \quad n \in \mathbb{N} ;
$$

здесь и далее

$$
\begin{gathered}
C_{n}=2^{-n} \pi^{(1-n) / 2}, \quad \beta=\frac{\sigma}{2}, \\
f_{\beta}(z ; \mu, \delta)= \begin{cases}\frac{2}{\Gamma(\mu / 2)} \int_{1}^{\infty} \phi(-\beta, \delta ;-z t)\left(t^{2}-1\right)^{\mu / 2-1} d t, & \mu>0, \\
\phi(-\beta, \delta ;-z), & \mu=0,\end{cases}
\end{gathered}
$$


где

$$
\phi(\gamma, \delta ; z)=\sum_{j=0}^{\infty} \frac{z^{n}}{j ! \Gamma(\gamma j+\delta)}, \quad \gamma>-1,
$$

- функция Райта (см. [28, 29]).

Приведем ряд утверждений, касающихся функции (20), которые нам понадобятся в дальнейшем. Доказательства можно найти в [7].

Лемма 4. Пусть $\zeta \in \mathbb{R}$. Функиия $\Gamma_{\sigma, n}(x-\xi, y-\eta)$ на множестве $\{|x-\xi|>0, \eta<y\}$, $\xi, x \in \mathbb{R}^{n}$, удовлетворяет уравнениюо

$$
\left(D_{\eta y}^{\sigma}-\Delta_{x}\right) D_{\eta y}^{\zeta} \Gamma_{\sigma, n}(x-\xi, y-\eta)=0
$$

как функиия переменных $x$ и у и уравнению

$$
\left(D_{y \eta}^{\sigma}-\Delta_{\xi}\right) D_{y \eta}^{\zeta} \Gamma_{\sigma, n}(x-\xi, y-\eta)=0
$$

как функиия переменных $\xi$ и $\eta$.

Введем следующие обозначения:

$$
E(z, \rho)=\exp \left(-\rho z^{1 /(1-\beta)}\right), \quad \gamma_{n}(z)= \begin{cases}1 & \text { при } n \leqslant 3 \\ |\ln z|+1 & \text { при } n=4, \\ z^{4-n} & \text { при } n \geqslant 5 .\end{cases}
$$

Лемма 5. Пусть $\zeta \in \mathbb{R}$. Справедливы неравенства

$$
\begin{aligned}
\left|D_{\eta y}^{\zeta} \Gamma_{\sigma, n}(x, y)\right| & \leqslant C y^{\beta(2-n)-\zeta-1} \gamma_{p}\left(|x| y^{-\beta}\right) E\left(|x| y^{-\beta}, \rho\right), \\
\left|\frac{\partial}{\partial x_{k}} D_{\eta y}^{\zeta} \Gamma_{\sigma, n}(x, y)\right| & \leqslant C\left|x_{k}\right| y^{-\beta n-\zeta-1} \gamma_{p+2}\left(|x| y^{-\beta}\right) E\left(|x| y^{-\beta}, \rho\right), \\
\left|\frac{\partial^{2}}{\partial x_{k}^{2}} D_{\eta y}^{\zeta} \Gamma_{\sigma, n}(x, y)\right| & \leqslant C y^{-\beta n-\zeta-1} \gamma_{q}\left(|x| y^{-\beta}\right) E\left(|x| y^{-\beta}, \rho\right),
\end{aligned}
$$

где

$$
p=\left\{\begin{array}{ll}
n, & \text { если } \zeta \in \mathbb{N} \cup\{0\}, \\
n+2, & \text { если } \zeta \notin \mathbb{N} \cup\{0\},
\end{array} \quad q= \begin{cases}n+2, & \text { если } \zeta \in \mathbb{N} \cup\{0\} \text { или } n=1, \\
n+4, & \text { если } \zeta \notin \mathbb{N} \cup\{0\} \text { и } n \geqslant 2,\end{cases}\right.
$$

$C=C(n, \sigma, \rho)$, а значение $\rho$ может быть выбрано любым из условия $\rho<(1-\beta) \beta^{\beta /(1-\beta)}$.

7. Функция Грина. Далее, если оператор $\mathbf{J}^{a, b}$ применяется к функции, зависящей от нескольких переменных, условимся писать $\mathbf{J}_{s, \theta}^{a, b}$, указывая в нижнем индексе переменные образа.

Рассмотрим функцию

$$
G(x, y, \xi, \eta)=\mathbf{J}_{x_{1}, \xi_{1}}^{a_{1}, b_{1}} \mathbf{J}_{x_{2}, \xi_{2}}^{a_{2}, b_{2}} \ldots \mathbf{J}_{x_{n}, \xi_{n}}^{a_{n}, b_{n}} \Gamma_{\sigma, n}(x, y-\eta)
$$

Далее будем обозначать $\Omega_{y}=\{(\xi, \eta): \xi \in S, \eta \in(0, y)\}$.

Сформулируем ряд свойств функции (26).

1. Функция (26) может быть представлена в виде

$$
G(x, y, \xi, \eta)=\Gamma_{\sigma, n}(x-\xi, y-\eta)+H(x, y, \xi, \eta),
$$

где $H(x, y, \xi, \eta)$ как функция переменных $x$ и $\xi$ (при фиксированных $y$ и $\eta, 0<\eta<y<T$ ) принадлежит пространству $C^{\infty}(\bar{S} \times S) \cap C^{\infty}(S \times \bar{S})$. Представление $(27)$ и гладкость функции $H(x, y, \xi, \eta)$ с учетом оценки (23) следуют из леммы 1.

2. Для любого $\zeta \in \mathbb{R}$ функция $H(x, y, \xi, \eta)$ на множестве $\Omega \times \Omega_{y}$ удовлетворяет уравнению

$$
\left(D_{\eta y}^{\sigma}-\Delta_{x}\right) D_{\eta y}^{\zeta} H(x, y, \xi, \eta)=0
$$

как функция переменных $x$ и $y$ и уравнению

$$
\left(D_{y \eta}^{\sigma}-\Delta_{\xi}\right) D_{y \eta}^{\zeta} H(x, y, \xi, \eta)=0
$$


как функция переменных $\xi$ и $\eta$. Равенства (28) и (29), с учетом неравенств (23), (24) и (25), а также леммы 3 , следуют из соотношений $(11),(12),(21)$ и (22).

3. Для любой фиксированных $(x, y) \in \Omega$ и $\eta<y$ справедливы соотношения

$$
\begin{aligned}
& \lim _{\xi_{k} \rightarrow a_{k}+} G(x, y, \xi, \eta)=0, \quad k \in K_{10} \cup K_{11}, \\
& \lim _{\xi_{k} \rightarrow b_{k}-} G(x, y, \xi, \eta)=0, \quad k \in K_{01} \cup K_{11} .
\end{aligned}
$$

Предельные соотношения (16) вместе с оценкой (23) дают (30) и (31).

4. Для любого $\zeta \in \mathbb{R}$ имеет место равенство

$$
\lim _{\eta \rightarrow y} D_{\eta y}^{\zeta} H(x, y, \xi, \eta)=0, \quad x \in S, \quad \xi \in S .
$$

Предельное соотношение (32) следует из (17) и (23).

Приведенные выше свойства позволяют назвать функцию $G(x, y, \xi, \eta)$ функцией Грина задачи (4), (5), (6) для уравнения (3).

8. Представление решения. В дальнейшем положим

$$
\mu_{j}=\sum_{i=0}^{j} \gamma_{i},
$$

где, как и раньше, $\gamma_{i}$ - элементы последовательности (2), с которой ассоциирован оператор дробного дифференцирования в уравнении (3).

Теорема 1. Пусть $и(x, y)$ - такое регулярное решение задачи (3)-(6), что $\partial u(x, y) / \partial x_{k} \in$ $L(\partial S \times(0, T))$, и для некоторого $\nu>0$ справедливы включения

$$
\begin{gathered}
y^{1-\mu} f(x, y) \in C(\bar{S} \times[0, T)), \\
y^{1-\mu} \varphi_{k}\left(x_{(k)}, y\right) \in C\left(\bar{S}_{(k)} \times[0, T)\right), \quad k \in K_{10} \cup K_{11} ; \\
y^{1-\mu} \psi_{k}\left(x_{(k)}, y\right) \in C\left(\bar{S}_{(k)} \times[0, T)\right), \quad k \in K_{01} \cup K_{11} ; \\
\tau_{j}(x) \in C(S), \quad j=0,1, \ldots, m-1 .
\end{gathered}
$$

Пусть такюе в случае $K_{00} \cup K_{01} \cup K_{10} \neq \emptyset$ выполнены предельные соотношения

$$
\begin{gathered}
\lim _{|x| \rightarrow \infty} \tau_{j}(x) \exp \left(-\rho|x|^{2 /(2-\sigma)}\right)=0, \quad j=0,1, \ldots, m-1, \\
\lim _{|x| \rightarrow \infty} y^{1-\mu} f(x, y) \exp \left(-\rho|x|^{2 /(2-\sigma)}\right)=0, \\
\lim _{|x| \rightarrow \infty} y^{1-\mu} u(x, y) \exp \left(-\rho|x|^{2 /(2-\sigma)}\right)=0,
\end{gathered}
$$

где $\rho<(1-\beta)(\beta / T)^{\beta /(1-\beta)}$. Тогда функиия $u(x, y)$ представима в виде

$$
\begin{aligned}
u(x, y)=\sum_{j=0}^{m-1} \int_{S} \tau_{j}(\xi)\left[D_{y \eta}^{\sigma-\mu_{j}} G(x, y, \xi, \eta)\right]_{\eta=0} d \xi+ & \\
& +\sum_{k \in K_{10} \cup K_{11}} \int_{0}^{y} \int_{S_{(k)}} \varphi_{k}\left(\xi_{(k)}, \eta\right)\left[\frac{\partial}{\partial \xi_{k}} G(x, y, \xi, \eta)\right]_{\xi_{k}=a_{k}} d \xi_{(k)} d y- \\
& -\sum_{k \in K_{01} \cup K_{11}} \int_{0}^{y} \int_{S_{(k)}} \psi_{k}\left(\xi_{(k)}, \eta\right)\left[\frac{\partial}{\partial \xi_{k}} G(x, y, \xi, \eta)\right]_{\xi_{k}=b_{k}} d \xi_{(k)} d y+ \\
& +\int_{0}^{y} \int_{S} f(\xi, \eta) G(x, y, \xi, \eta) d \xi d \eta
\end{aligned}
$$


Доказательство. Рассмотрим сначала случай, когда $K_{00} \cup K_{01} \cup K_{10}=\emptyset$ (т.е. область $S$ ограничена). В силу (30) и (31) имеем

$$
\left.G(x, y, \xi, \eta)\right|_{(\xi, \eta) \in \partial S \times(0, T)}=0 .
$$

Кроме того, из (22), (27) и (29) следует, что

$$
\left(D_{y \eta}^{\sigma}-\Delta_{\xi}\right) G(x, y, \xi, \eta)=0, \quad(\xi, \eta) \in S \times(0, y) .
$$

Таким образом, формула (36) следует из общего представления решения диффузионно-волнового уравнения (см. [7, теорема 1]).

Пусть теперь $K_{00} \cup K_{01} \cup K_{10} \neq \emptyset$, т.е. область $S$ содержит бесконечную компоненту. Для положительного $R$ обозначим $n$-мерный куб (декартово произведение интервалов $-R<x_{k}<R$ ) через $I_{R}=(-R, R) \times \cdots \times(-R, R)$ и

$$
S_{R}=S \cap I_{R}
$$

Обозначим через $S_{(k), R}$ проекцию вдоль $x_{k}$ части границы области $S_{R}$, которая при больших $R$ остается внутри $S$ (это будет иметь место для индексов $k \in K_{10} \cup K_{01} \cup K_{11}$ ). Кроме того, символом $G_{R}(x, y, \xi \eta)$ будем обозначать функцию Грина, соответствующую области $S_{R}$, которая, очевидно, ограничена. Поэтому, с учетом вышесказанного, для любых $x \in S_{R}$ и $y \in(0, R)$ имеет место соотношение

$$
\begin{aligned}
u(x, y)=\sum_{j=0}^{m-1} \int_{S_{R}} \tau_{j}(\xi)\left[D_{y \eta}^{\sigma-\mu_{j}} G_{R}(x, y, \xi, \eta)\right]_{\eta=0} d \xi+ & \\
& +\sum_{k \in K_{10} \cup K_{11}} \int_{0}^{y} \int_{S_{(k)}} \varphi_{k}\left(\xi_{(k)}, \eta\right)\left[\frac{\partial}{\partial \xi_{k}} G_{R}(x, y, \xi, \eta)\right]_{\xi_{k}=a_{k}} d \xi_{(k)} d y- \\
& -\sum_{k \in K_{01} \cup K_{11}} \int_{0}^{y} \int_{S_{(k)}} \psi_{k}\left(\xi_{(k)}, \eta\right)\left[\frac{\partial}{\partial \xi_{k}} G_{R}(x, y, \xi, \eta)\right]_{\xi_{k}=b_{k}} d \xi_{(k)} d y+ \\
& +\int_{0}^{y} \int_{S_{R}} f(\xi, \eta) G_{R}(x, y, \xi, \eta) d \xi d \eta+\Phi_{k, R}(x, y)+\Psi_{k, R}(x, y)
\end{aligned}
$$

где

$$
\begin{aligned}
\Phi_{k, R}(x, y) & =\sum_{k \notin K_{10} \cup K_{11}} \int_{0}^{y} \int_{S_{(k), R}}\left[u(\xi, \eta) \frac{\partial}{\partial \xi_{k}} G_{R}(x, y, \xi, \eta)\right]_{\xi_{k}=-R} d \xi_{(k)} d y \\
\Psi_{k, R}(x, y) & =-\sum_{k \notin K_{01} \cup K_{11}} \int_{0}^{y} \int_{S_{(k), R}}\left[u(\xi, \eta) \frac{\partial}{\partial \xi_{k}} G_{R}(x, y, \xi, \eta)\right]_{\xi_{k}=R} d \xi_{(k)} d y
\end{aligned}
$$

Равенство (37) справедливо для любого достаточно большого $R$. В силу (24) и (35)

$$
\lim _{R \rightarrow \infty} \Phi_{k, R}(x, y)=0, \quad \lim _{R \rightarrow \infty} \Psi_{k, R}(x, y)=0 .
$$

Поэтому, устремляя $R$ к бесконечности, учитывая (23), (24), (33) и (34), приходим к (36).

Заметим, что из теоремы 1 , вообще говоря, не следует, что любая функция вида (36) является регулярным решением задачи (3)-(6). Чтобы это имело место, необходимы дополнительные условия на гладкость правой части и функций в начальных и краевых условиях, а также на распределение параметров $\gamma_{k}$ (см. [7]). Однако из представления (36) следует единственность решения рассматриваемой задачи. При этом в случае $K_{00} \cup K_{01} \cup K_{10} \neq \emptyset$ (т.е. если область $S$ не 
ограничена), единственность решения имеет место в классе функций, удовлетворяющих аналогу условия А. Н. Тихонова:

$$
\lim _{|x| \rightarrow \infty} y^{1-\mu} u(x, y) \exp \left(-\omega|x|^{2 /(2-\sigma)}\right)=0
$$

для некоторого $\omega>0$.

\section{СПИСОК ЛИТЕРАТУРЫ}

1. Вагабов А. И. Представление решения первой краевой задачи для уравнения теплопроводности в виде интегралов Пуассона и их приложения// Докл. РАН. - 2000. - 375, № 4. - С. 439-442.

2. Ворошилов A. А., Килбас A. А. Задача Коши для диффузионно-волнового уравнения с частной производной Капуто// Диффер. уравн. - 2006. - 42, № 5. - С. 599-609.

3. Джрбашян M. М., Нерсесян А. Б. Дробные производные и задачи Коши для дифференциальных уравнений дробного порядка// Изв. акад. наук Арм. ССР. - 1968. - 3, № 1. - С. 3-28.

4. Кочубей A. Н. Диффузия дробного порядка// Диффер. уравн. - 1990. - 26, № 4. - С. 660-670.

5. Нахушев А. М. Дробное исчисление и его применение. - М.: Физматлит, 2003.

6. Псху A. В. Решение краевой задачи для уравнения с частными производными дробного порядка// Диффер. уравн. - 2003. - 39, № 8. - С. 1092-1099.

7. Псху А. В. Фундаментальное решение диффузионно-волнового уравнения дробного порядка// Изв. PAH. Cер. мат. - 2009. - 73, № 2. - C. 141-182.

8. Псху А. В. Первая краевая задача для дробного диффузионно-волнового уравнения в нецилиндрической области// Изв. РАН. Сер. мат. - 2017. - 81, № 6. - С. 158-179.

9. Псху A. В. Уравнения в частных производных дробного порядка. - М.: Наука, 2005.

10. Псху A. В. Решение первой краевой задачи для уравнения диффузии дробного порядка// Диффер. уравн. - 2003. - 39, № 9. - С. 1286-1289.

11. Псху А. В. Решение краевых задач для уравнения диффузии дробного порядка методом функции Грина// Диффер. уравн. - 2003. - 39, № 10. - С. 1430-1433.

12. Самко С. Г., Килбас А. А., Маричев О. И. Интегралы и производные дробного порядка и некоторые их приложения. - Минск: Наука и техника, 1987.

13. Тарасов B. E. Модели теоретической физики с интегро-дифференцированием дробного порядка. М.-Ижевск: Ин-т комп. исслед., 2011.

14. Agrawal O. P. Solution for a fractional diffusion-wave equation defined in a bounded domain// Nonlin. Dynam. - 2002. - 29, № 1. - P. 145-155.

15. Atanacković T. M., Pilipović S., Stanković B., Zorica D. Fractional Calculus with Applications in Mechanics. - London: Wiley, 2014.

16. Eidelman S. D., Kochubei A. N. Cauchy problem for fractional diffusion equations// J. Differ. Equ. — 2004. - 199. - P. 211-255.

17. Fujita $Y a$. Integrodifferential equation which interpolates the heat equation and the wave equation, I// Osaka J. Math. - 1990. — 27, № 2. - P. 309-321.

18. Fujita $Y a$. Integrodifferential equation which interpolates the heat equation and the wave equation, II// Osaka J. Math. - 1990. — 27, № 4. - P. 797-804.

19. Gorenflo R., Iskenderov A., Luchko Yu. Mapping between solutions of fractional diffusion-wave equations// Fract. Calcul. Appl. Anal. - 2000. - 3, № 1. - P. 75-86.

20. Kilbas A. A., Srivastava H. M., Trujillo J. J. Theory and Applications of Fractional Differential Equations. - Amsterdam: North-Holland, 2006.

21. Mainardi F. The fundamental solutions for the fractional diffusion-wave equation// Appl. Math. Lett. 1996. - 9, № 6. - P. 23-28.

22. Mainardi F. Fractional Calculus and Waves in Linear Viscoelasticity: An Introduction to Mathematical Models. - Singapore: World Scientific, 2010.

23. Miller K., Ross B. An Introduction to the Fractional Calculus and Fractional Differential Equation. - New York: Wiley, 1993.

24. Oldham K., Spanier J. Fractional Calculus. — New York: Academic Press, 1974.

25. Podlubny I. Fractional Differential Equations. - San Diego-New York-London: Academic Press, 1999.

26. Schneider W. R., Wyss W. Fractional diffusion and wave equations// J. Math. Phys. — 1989. — 30, № 1 . - P. 134-144. 
27. Uchaikin V. V. Fractional Derivatives for Physicists and Engineers. - Berlin-Beijing: Springer, 2013.

28. Wright E. M. On the coefficients of power series having exponential singularities// J. London Math. Soc. - 1933. - 8, № 29. - P. 71-79.

29. Wright E. M. The generalized Bessel function of order greater than one// Quart. J. Math. Oxford Ser. 1940. - 11. - P. 36-48.

30. Wyss W. The fractional diffusion equation// J. Math. Phys. - 1986. - 27, № 11. - P. 2782-2785.

Псху Арсен Владимирович

Институт прикладной математики и автоматизации,

Кабардино-Балкарский научный центр Российской академии наук, Нальчик

E-mail: pskhu@list.ru 